\title{
A pilot study differentiating recurrent major depression from bipolar disorder cycling on the depressive pole
}

This article was published in the following Dove Press journal:

Neuropsychiatric Disease and Treatment

II November 2010

Number of times this article has been viewed

Marty Hinz'

Alvin Stein ${ }^{2}$

Thomas Uncini ${ }^{3}$

'Clinical Research, NeuroResearch Clinics, Inc., Cape Coral, FL, USA;

${ }^{2}$ Stein Orthopedic Associates, Plantation, FL, USA; ${ }^{3}$ DBS Labs, Duluth, MN, USA
Correspondence: Marty Hinz Clinical Research, Neuro Research Clinics, Inc., 1008 Dolphin Drive, Cape Coral, FL 33904, USA $\mathrm{Tel}+\mathrm{I} 2183100730$

Fax +I 2186261638

Email marty@hinzmd.com
Purpose: A novel method for differentiating and treating bipolar disorder cycling on the depressive pole from patients who are suffering a major depressive episode is explored in this work. To confirm the diagnosis of type 1 or type 2 bipolar disorder, the Diagnostic and Statistical Manual of Mental Disorders (DSM-IV) criteria require that at least one manic or hypomanic episode be identified. History of one or more manic or hypomanic episodes may be impossible to obtain, representing a potential blind spot in the DSM-IV diagnostic criteria. Many bipolar patients who cycle primarily on the depressive side for many years carry a misdiagnosis of recurrent major depression, leading to treatment with antidepressants that achieve little or no relief of symptoms. This article discusses a novel approach for diagnosing and treating patients with bipolar disorder cycling on the depressive pole versus patients with recurrent major depression.

Patients and methods: Patients involved in this study were formally diagnosed with recurrent major depression under DSM-IV criteria and had no medical history of mania or hypomania to support the diagnosis of bipolar disorder. All patients had suffered multiple depression treatment failures in the past, when evaluated under DSM-IV guidelines, secondary to administration of antidepressant drugs and/or serotonin with dopamine amino acid precursors.

Results: This study contained 1600 patients who were diagnosed with recurrent major depression under the DSM-IV criteria. All patients had no medical history of mania or hypomania. All patients experienced no relief of depression symptoms on level 3 amino acid dosing values of the amino acid precursor dosing protocol. Of 1600 patients studied, 117 (7.3\%) nonresponder patients were identified who experienced no relief of depression symptoms when the serotonin and dopamine amino acid precursor dosing values were adjusted to establish urinary serotonin and urinary dopamine levels in the Phase III therapeutic ranges. All of the 117 nonresponders who achieved no relief of depression symptoms were continued on this amino acid dosing value, and a mood-stabilizing drug was started. At this point, complete relief of depression symptoms, under evaluation with DSM-IV criteria, was noted in 114 patients within 1-5 days. With further dose adjustment of the mood-stabilizing drug, the remaining three nonresponders achieved relief of depression symptoms.

Conclusion: Resolution of depression symptoms with the addition of a mood-stabilizing drug in combination with proper levels of serotonin and dopamine amino acid precursors was the basis for a clinical diagnosis of bipolar disorder cycling on the depressive pole.

Keywords: depression, bipolar, serotonin, dopamine, mania, hypomania

\section{Introduction}

In order to make the diagnosis of type 1 or type 2 bipolar disorder under Diagnostic and Statistical Manual of Mental Disorders (DSM-IV) guidelines, the patient's medical 
history must include one or more manic or hypomanic episodes, respectively. ${ }^{1}$ The history of mania or hypomania may be obscure or nonexistent. For example, the bipolar patient may be cycling heavily on the depressive pole with the last manic or hypomanic episode having occurred many years ago. This episode may have lasted for only 2 weeks, during which time neither the patient nor others around the patient ever appreciated its presence. This obscured medical history represents a potential blind spot in the DSM-IV criteria for diagnosing bipolar disorder cycling on the depressive pole. It is not uncommon in patients with bipolar disorder cycling on the depressive pole, while looking for relief of depression symptoms, to get caught in a seemingly endless cycle of shopping for health care providers. These physicians may have prescribed most or all of the antidepressants medically available for treatment without getting complete relief of depression symptoms. This potential blind spot in the DSM-IV criteria leads to a misdiagnosis of recurrent major depression.

The novel approach described in this writing requires the administration of serotonin and dopamine amino acid precursors with cofactors to reach the Phase III therapeutic ranges (herein referred to as the target ranges) as guided by the use of urinary serotonin and urinary dopamine organic cation transporter (OCT) functional status determination (herein referred to as 'OCT assay interpretation'). ${ }^{2-5}$

The basis for the OCT assay interpretation model requires two or more serial urinary serotonin and dopamine assays while taking varied amino acid precursor daily dosing values. Results of two or more assays are then compared in order to determine the change in urinary serotonin and dopamine levels in response to the change in dosing. A urinary serotonin or dopamine value $<80$ or $475 \mu \mathrm{g}$ of monoamine per gram of creatinine, respectively, indicates Phase II responses. A urinary serotonin or dopamine value $>80$ or $475 \mu \mathrm{g}$ of monoamine per gram of creatinine, respectively, is interpreted as being in Phase I or Phase III. Differentiation of Phase I from Phase III is a follows. If a direct correlation is found between amino acid dosing and urinary assay response, it is referred to as a Phase III response. An inverse correlation is referred to as a Phase I response. The Phase III therapeutic range for urinary serotonin is defined as 80-240 $\mu \mathrm{g}$ of serotonin per gram of creatinine. The Phase III therapeutic range for urinary dopamine is defined as 475-1100 $\mu \mathrm{g}$ of dopamine per gram of creatinine. ${ }^{2-5}$

Peer-reviewed scientific publications discussing urinary serotonin and urinary dopamine phase analysis under the
OCT model were published in $2009^{2,4}$ and $2010 .^{3,5}$ These publications outlined the mechanisms of the "three-phase model' in connection with urinary serotonin and urinary dopamine under a novel renal transporter model. This transporter model potentially describes the etiology of the 'three-phase response' in monoamine assays during the administration of varied amino acid precursor daily dosing values. ${ }^{3}$ Urinary serotonin and dopamine levels are primarily dependent upon the interaction of the basolateral monoamine transporters with the apical monoamine transporters of the proximal convoluted renal tubule cells of the kidneys. ${ }^{3}$

Most notable with this novel approach is the ability to differentiate patients with bipolar disorder cycling on the depressive pole from patients suffering from recurrent major depression, and then implement effective treatment.

\section{Material and methods}

Processing, management, and assay of the urine samples collected for this study were as follows. Urine samples were collected $6 \mathrm{~h}$ prior to bedtime with 4:00 PM being the most frequent collection time point. The samples were stabilized in $6 \mathrm{~N} \mathrm{HCl}$ to preserve urinary dopamine and urinary serotonin. The urine samples were collected after a minimum of 1 week during which the patient was taking a specific daily dosing of amino acid precursors of serotonin and dopamine where no doses were missed. Samples were shipped to DBS Laboratories (Duluth, $\mathrm{MN}$ ), which is operated under the direction of one of the authors (Thomas Uncini, MD, hospital-based pathologist, dual board certified in laboratory medicine and forensic pathology). Urinary dopamine and serotonin were assayed utilizing commercially available radioimmunoassay kits (3 CAT RIA IB88501 and IB89527; Immuno Biological Laboratories, Inc., Minneapolis, MN). The DBS laboratory is accredited as a high complexity laboratory by Clinical Laboratory Improvement Amendments to perform these assays. ${ }^{3,6}$

\section{The protocol}

The protocol for treatment of depression consisted of the amino acid dosing values found in Table 1. This protocol was covered in previous peer-reviewed literature. ${ }^{2}$

The initial step of the protocol was the administration of serotonin and dopamine amino acid precursors with no OCT assay interpretation. Three dosing levels were available as noted in Table 1. At the first visit, patients were started on level 1 amino acid dosing. Patients were then seen weekly for follow-up clinic visits. 
Milligrams 5-HTP/Milligrams L-Tyrosine

\begin{tabular}{|c|c|c|c|c|}
\hline AM & NOON & 4 PM & 7 PM & \\
\hline $150 / 1,500$ & ----- & $150 / 1,500$ & ---- & Level 1 \\
\hline $150 / 1,500$ & $150 / 1,500$ & $300 / 1,000$ & ---- & Level 2 \\
\hline $150 / 1,500$ & $150 / 1,500$ & $300 / 1,000$ & $300 / 1,000$ & Level 3 \\
\hline
\end{tabular}

Table I Amino acid precursor dosing protocol. Subjects also received the following daily dosing values of cofactors: I) $1000 \mathrm{mg}$ vitamin C, 2) $220 \mathrm{mg}$ calcium citrate, 3) 75 mg vitamin B6, and 4) $400 \mu \mathrm{g}$ folate. Copyright $\odot$ 2009, CRC Press. Adapted with permission from Hinz M. Depression. In: Kohlstadt l, editor. Food and Nutrients in Disease Management. Boca Raton, FL: CRC Press; 2009:465-48I.

The question to be answered in evaluating patients after 1 week of taking a specific amino acid dosing value was 'What was the status of the depression symptoms yesterday?' Since it takes up to 5 days for the maximum benefit of an amino acid dosing change to be seen, secondary to equilibration of the amino acids, results from the day before the visit were found to be more reliable than inquiring about the status of depression symptom for the entire week.

Since the maximum benefit of any dosing change occurs within 5 days, there is no purpose in waiting longer than 1 week to see if an amino acid dosing change achieved additional relief of symptoms. This only increases the amount of time needed to find the proper dose leading to resolution of the symptoms. ${ }^{7}$

If there was no relief of depression symptoms at the weekly visit, the amino acid dosing was adjusted upward to the next dosing level (level 2 or level 3). The goal was to obtain relief of depression symptoms or reach level 3 amino acid dosing with no relief of symptoms, whichever occurred first. ${ }^{2}$

At the initial visit, all prescription drugs were continued. Prescription antidepressant drugs were continued until full relief of depression symptoms was obtained, and then tapered to a stop, at the option of the caregiver. It was noted that drugs may require being stopped sooner if drug side effects emerge. As neurotransmitter levels increase with amino acid precursor administration, drug side effects may occur in $\sim 5 \%$ of patients. The emergence of drug side effects may be a source of confusion for the caregiver. Since the last thing changed in the patient's treatment plan was the amino acid dosing, there is a tendency to focus on the amino acids as the source of side effects that were actually due to prescription drug toxic side effects.

Failure to achieve relief of depression symptoms, on evaluation with DSM-IV guidelines, after 1 week of taking level 3 amino acid dosing of Table 1 was the indication for initiation of OCT assay interpretation studies to guide further amino acid precursor dosing value changes.
Subsequent to the interpretation of each urine sample collected, the amino acid dosing was adjusted in response to OCT assay interpretation findings. This was focused on achieving both the urinary serotonin and dopamine in the Phase III therapeutic ranges (the target ranges). The end point of collecting samples for OCT assay interpretation was whichever came first of the following: i) resolution of depression symptoms, ii) obtaining both the urinary serotonin and dopamine in the target ranges, or iii) the patient dropping out of treatment.

If no relief of depression symptoms was observed with the urinary serotonin and dopamine in the target ranges, the amino acids were continued at that dosing value and a mood-stabilizing drug was added. The choice of mood-stabilizing drug was either lithium carbonate $300 \mathrm{mg}$ twice a day or divalprex sodium $250 \mathrm{mg} 3$ times a day at the caregiver's discretion.

L-Dopa and L-tyrosine have an ability to deplete sulfur amino acids. Based on previous experience and peerreviewed literature, L-cysteine was added to the serotonin and dopamine amino acid precursors in the amounts of $4500 \mathrm{mg} /$ day in adults in divided doses. ${ }^{2}$ Selenium $400 \mathrm{mcg} /$ day was administered with the L-cysteine to address concerns raised in the literature regarding L-cysteine facilitating neurotoxic insult by methylmercury. ${ }^{8}$ Other literature notes that selenium irreversibly binds to methylmercury rendering it nontoxic. ${ }^{9}$

\section{Results}

Patients selected had been formally diagnosed with depression under DSM-IV criteria and carried no previous diagnosis or medical history supporting bipolar disorder.

The group studied consisted of 1600 patients diagnosed with recurrent major depression who failed to respond to treatment with amino acid precursors at the level 3 dosing (outlined in Table 1) on evaluation under DSM-IV guidelines. These 1600 patients had urine samples collected, and OCT 
assay interpretation was performed with amino acid dosing adjustments focused on achieving urinary serotonin and dopamine in the target ranges.

The status of depression symptoms was evaluated with the DSM-IV criteria at each visit. Of the 1600 patients starting the OCT assay interpretation, the following three groupings of patients ultimately were defined: i) patients who achieved relief of symptoms in response to amino acid precursor dosing value adjustment guided by OCT assay interpretation, ii) patients who achieved no relief of symptoms with the amino acid precursor dosing required for achieving urinary serotonin and dopamine in the target ranges, and iii) patients who dropped out of treatment.

Patients achieving relief of symptoms with amino acid dose adjustment and patients dropping out were not tracked in this study. The goal of the study was to define a group of patients who were nonresponders with urinary serotonin and dopamine in the target ranges. Of the initial starting group of $\mathrm{N}=1600$, a group of $\mathrm{N}=117(7.3 \%)$ was determined to be nonresponders.

Demographics of the 117 nonresponders were as follows. There were 73 females in the nonresponder group $(62.4 \%)$. There were 44 males in the nonresponder group (37.6\%). The age range for the entire nonresponder group was 18.3-82.9 with a mean age of 55.2 and a standard deviation of 13.2 years. The age range for the female nonresponder group $(\mathrm{N}=73)$ was $18.3-75.3$ with a mean of 53.8 and a standard deviation of 11.9 years. The age range for the male nonresponder group $(\mathrm{N}=44)$ was $25.0-82.9$ with a mean of 55.2 and a standard deviation of 13.2 years.

Nonresponders were continued on the amino acid dosing values needed to achieve the target ranges and a mood-stabilizing drug was started. The choice and dose of the mood-stabilizing drugs were lithium carbonate $300 \mathrm{mg}$ twice a day or divalprex sodium $250 \mathrm{mg} 3$ times a day, at the caregiver's discretion.

Of the 117 patients started on a mood-stabilizing drug in combination with the amino acid dosing needed to establish the target ranges, 114 achieved full relief of depression symptoms within 1-5 days of starting the drug. Of the three patients who did not respond when the initial dose of the mood-stabilizing drug was added, further adjustment of the mood-stabilizing drug was guided by serum assays of the drug levels with the goal of establishing lithium or valproic acid serum levels in the therapeutic range. During the process of serum-guided adjustment of the mood-stabilizing drug, relief of depression symptoms was obtained in the final three patients. A positive response to adding the moodstabilizing drug to the amino acid dosing of the target ranges was the basis for a clinical diagnosis of bipolar disorder cycling on the depressive pole. The type was considered undifferentiated since no history of mania or hypomania existed.

The conclusion was that $100 \%$ of patients in whom a mood-stabilizing drug was administered in conjunction with amino acid precursors were clinically diagnosed with bipolar disorder cycling on the depressive pole, while experiencing complete relief of depression symptoms. A significant point is the dramatic response of these patients to the starting dose of the mood-stabilizing drug. Up until initiation of the mood-stabilizing drug, none of the patients had experienced any relief or improvement of depression symptoms. The starting dose of the mood-stabilizing drugs was well tolerated with no reported start-up problems.

The choice of which mood-stabilizing drug to prescribe was at the discretion of the caregiver; $71 \%$ of patients were treated with lithium carbonate, and $29 \%$ of patients were treated with divalprex sodium. Analysis of results revealed no significant difference in outcomes with the mood-stabilizing drug selected. Both mood-stabilizing drugs appear to be equally effective with all patients achieving relief of depression symptoms due to bipolar disorder cycling on the depressive pole.

Review of group amino acid dosing values, where both the urinary serotonin and dopamine were in the target ranges, revealed daily dosing values were highly individualized with no standard dosing apparent. In the group of 117 nonresponders to amino acids alone, the group amino acid dosing values in the target ranges were as follows.

The group 5-HTP dosing range was 37.5-1800 mg/day with a mean of $300 \mathrm{mg} /$ day and a standard deviation of $380.5 \mathrm{mg} /$ day. L-Tyrosine group dosing range was 2500-13,000 mg/day with a mean of $7000 \mathrm{mg} /$ day and a standard deviation of $2148.5 \mathrm{mg} /$ day. L-Dopa group dosing range was 0-2940 $\mathrm{mg}$ /day with a mean of $240 \mathrm{mg} /$ day and a standard deviation of $285.8 \mathrm{mg} /$ day. $^{6}$

Treatment time to stabilization was as follows. All patients at the start of the study had undergone 3 weeks of treatment utilizing the amino acid dosing value adjustment under the protocol of Table 1 . Time to achieve urinary serotonin and dopamine in the Phase III therapeutic ranges beyond level 3 dosing was 2-12 weeks with a mean of 6 weeks and a standard deviation of 2.23 weeks. In 114 of the patients started on a mood-stabilizing drug, one additional week of treatment time was required to achieve relief of symptoms. The average 
time of treatment from start of the amino acids in Table 1 to relief of symptoms with the mood-stabilizing drugs was 10 weeks with a range of 6-16 weeks.

Physicians involved in this study reported no relapse of depression symptoms as long as patients were compliant with treatment prescribed. The longest follow-up period for a patient in this study is currently 8 years.

\section{Discussion}

The clinical diagnosis of depression was made in the primary care setting. It was indicated that the diagnosis of depression had been made under DSM-IV criteria. There were no reports of structure interviews being performed in these practices which may represent a limitation of this depression care.

While the approach of administering L-tyrosine with L-dopa may seem counterintuitive, the rationale for its use is supported by the literature and the research experience leading up to this article. Peer-reviewed literature notes administering L-dopa without proper levels of L-tyrosine can lead to significant fluctuations in urinary dopamine levels. Dopamine fluctuations interfere with OCT assay interpretation, leading to inconsistent results. Dopamine fluctuations can also decrease the efficacy of L-dopa, as the clinical response fluctuates. ${ }^{4}$ It is also known that administration of L-dopa can lead to depletion of L-tyrosine. ${ }^{10}$

The effects of mood-stabilizing drugs (lithium carbonate or divalprex sodium) used in this study appear to be potentiated by the serotonin and dopamine amino acid precursor in dosing values needed to establish the target ranges. There appears to be a synergy. The mechanism of this potential synergy is unknown. In this pilot study, patients who were diagnosed with bipolar disorder cycling on the depressive pole experienced no relief of symptoms in the past although mood-stabilizing drugs were administered at various dosing values including levels verified as therapeutic by serum assays. In this study, they obtained relief of symptoms predominantly on the starting dose of drug when added to amino acid precursors at dosing values required to establish the target ranges.

The following patient profile exists for those patients newly diagnosed in this study with bipolar disorder cycling on the depressive pole under this protocol. The typical patient has a history of being treated with antidepressants for recurrent major depression for many years without relief of depression symptoms. Once symptoms of depression are under control, with the addition of a mood-stabilizing drug, it is not uncommon for patients to report that their symptoms have been present since high school or earlier in life. Patient reports of suffering since high school are common and even more impressive when it is realized that $80 \%$ of the patients in this study ranged from 40 to 65 years of age, meaning years of suffering without effective treatment.

Over the years, these patients have seen many physicians while looking for relief of depression symptoms and have taken most or all of the antidepressants available without complete relief of symptoms. ${ }^{2}$ It is suggested that future studies of this protocol which differentiates bipolar depression from recurrent major depression should incorporate the following screening in order to generate a group with a higher percentage of previously undiagnosed bipolar disorder cycling on the depressive pole. i) A history of depression for over 10 years. ii) A history of having seen five or more caregivers for treatment of depression. iii) A history of having taken five or more antidepressant drugs in the past without full relief of symptoms. It is also suggested that in patients who meet this criteria, the amino acid dosing simply be started on level 1 dosing of Table 1 and then a urine sample be obtained in 1 week and submitted for OCT assay interpretation. This will decrease the time of treatment by 3 weeks.

As noted previously, when this protocol was initiated, any prescription drugs being taken were continued. Once the patient with bipolar disorder cycling on the depressive pole achieves relief of depression symptoms and the diagnosis of bipolar disorder cycling on the depressive pole is made, any antidepressants, which are not indicated for monotherapy for treatment with bipolar depression under US Food and Drug Administration (FDA) guidelines, should be stopped. , $7,11,12^{2}$ However, it was found that many of these patients, finally symptom free for the first time in years, are hesitant or even highly resistant to giving up anything in their treatment plan that has finally gotten them relief of symptoms, including the antidepressants. These feelings on the part of the patient may be exceptionally strong. An effective approach to eliminating the antidepressants that are not indicated for monotherapy in bipolar depression from the treatment plan, in the face of strong patient resistance, is to simply wait 2-3 months after relief of symptoms and then revisit the issue of slowly tapering the antidepressants to a stop.

Implementation of this protocol is time intensive. The amino acid dosing value OCT assay interpretation cycle is 2 weeks. When the amino acid dosing value is 
changed, it takes 1 week before urine can be collected in the steady state, and then it takes another week to get the test results of OCT assay interpretation reported back to the physician in order to prescribe the next amino acid dosing value. Patients at initial visit orientation and on follow-up visits need to be prepared for the longest treatment time possible (2-4 months), although some patients may find relief of symptoms in 1-2 weeks. This is done to prevent patients from dropping out of treatment due to perceived lack of results after several weeks. Response time under this approach varies greatly. Since there is no way of predicting at which point in this process the patient will achieve relief of symptoms, all patients need to be properly oriented at the first visit to the indeterminate length of time with reinforcement of this orientation at subsequent visits. While weekly visits over a 4-month period may seem like a long time to get relief of symptoms, in fact, most bipolar disorder cycling on the depressive pole have suffered with disease symptoms for 20-40 years or longer and the time investment is relatively small compared to the prolonged length of suffering and the cost due to ineffectiveness of previous medical care.

As treatment under this protocol progresses, most patients report no relief of depression symptoms until the proper amino acid dosing or amino acid dosing with mood-stabilizer dosing has been implemented. It was relatively rare for patients to achieve gradual relief of symptoms from visit to visit. Resolution of symptoms in patients can be and often is dramatic and abrupt, analogous to a light switch being either off (with symptoms) or on (without symptoms). It was not uncommon for a patient who had been under treatment for many weeks to return for a clinic visit and report the exact day that relief of depression symptoms occurred. Waiting for this dramatic effect to occur without the patient understanding the process involved may lead to an increased drop-out rate of patients prior to relief of symptoms. For example, the patient who has been under treatment for many weeks, with no improvement of depression, may contemplate dropping out of treatment when, in fact, they are on the doorstep of dramatic improvement.

\section{Conclusion}

The novel approach of this pilot study clinically differentiates recurrent major depression from bipolar disorder cycling on the depressive pole. Although this approach appears to be effective in treatment of bipolar disorder cycling on the depressive pole, more studies are needed.
Bipolar disorder cycling on the depressive pole is frequently misdiagnosed by caregivers. One of the primary stumbling blocks is the inability to elicit a proper medical history of one or more manic or hypomanic episodes in the patient's past in order to satisfy the DSM-IV criteria. This potential blind spot in the DSM-IV criteria leads to antidepressants being prescribed to patients with bipolar depression, a practice that is specifically not indicated as monotherapy under FDA guidelines. ${ }^{6,7,11,12}$

The protocol of this study has been in use since 2004 with no reported failures in the treatment of bipolar disorder cycling on the depressive pole when the protocol was followed properly. This is a pilot study. The intent of this article is to disseminate some of the knowledge gained in this research, spark interest leading to more research, refine the protocol with more studies, and elicit scrutiny of these observations.

\section{Disclosure}

Marty Hinz and Thomas Uncini are owner and medical director of DBS Labs, respectively, Duluth, MN, USA. Alvin Stein reports no disclosures.

\section{References}

1. American Psychiatric Association. Diagnostic and Statistical Manual of Mental Disorder IV (DSM IV). 4th ed. Washington, DC: American Psychiatric Association;1994:327-352.

2. Hinz M. Depression. In: Kohlstadt I, editor. Food and Nutrients in Disease Management. Boca Raton, FL: CRC Press. 2009:465-481.

3. Hinz M, Stein A, Uncini T. The dual-gate lumen model of renal monoamine transport. Neuropsychiatr Dis Treat. 2010;6(1):387-392.

4. Trachte GJ, Uncini T, Hinz M. Both stimulatory and inhibitory effects of dietary 5-hydroxytryptophan and tyrosine are found on urinary excretion of serotonin and dopamine in a large human population. Neuropsychiatr Dis Treat. 2009;5:227-235.

5. Hinz M, Stein A, Trachte G, Uncini T. Comprehensive analysis of urinary neurotransmitter testing. Open Access Journal of Urology. 2010; In press.

6. Wyeth Pharmaceuticals, Inc. Effexor XR(r) (velafaxine hydrochloride) Extended-Release Capsules. Prescribing information. July 2009. Philadelphia, PA: Wyeth Pharmaceuticals, Inc. Available from: http://www.wyeth.com/content/showlabeling.asp?id=100. Accessed 2010 May 12.

7. Forest Labs. Lexapro(r) (escitalopram oxalate) prescribing information. Jan 2009. St. Louis, MO: Forest Labs. Available from: http://www.frx. com/pi/lexapro_pi.pdf. Accessed 2010 Jun 2.

8. Spindle A, Matsumoto N. Enhancement of methylmercury toxicity by L-cystine in cultured mouse blastocysts. Reprod Toxicol. 1987-1988;1(4):279-284.

9. Fair PH, Dougherty WJ, Braddon SA. Methyl mercury and selenium interaction in relation to mouse kidney gamma-glutamyltranspeptidase, ultrastructure, and function. Toxicol Appl Pharmacol. 1985;80(1): 78-96.

10. Karobath M, Diaz JL, Huttunen MO. The effect of L-dopa on the concentrations of tryptophan, tyrosine, and serotonin in rat brain. Eur J Pharmacol. 1971;14(4):393-396. 
11. GlaxoSmithKline. Wellbutrin XL(r) (bupropion hydrochloride extended-release tablets). Dec 2008. Research Triangle Park, NC: Glaxo SmithKline. Available from: http://us.gsk.com/products/assets/ us_wellbutrinXL.pdf. Accessed 2010 Jun 2.
12. Eli Lilly and Company. Prozac (fluoxetine hydrochloride) prescribing information. Oct 2009. Indianapolis (IN): Eli Lilly and Company. Available from: http://pi.lilly.com/us/prozac.pdf. Accessed 2010 Jun 2.

\section{Publish your work in this journal}

Neuropsychiatric Disease and Treatment is an international, peerreviewed journal of clinical therapeutics and pharmacology focusing on concise rapid reporting of clinical or pre-clinical studies on a range of neuropsychiatric and neurological disorders. This journal is indexed on PubMed Central, the 'PsycINFO' database and CAS, and is the official

Submit your manuscript here: http://www.dovepress.com/neuropsychiatric-disease-and-treatment-journal journal of The International Neuropsychiatric Association (INA). The manuscript management system is completely online and includes a very quick and fair peer-review system, which is all easy to use. Visit http://www.dovepress.com/testimonials.php to read real quotes from published authors. 